\title{
Qualidade de vida e ansiedade relacionadas às alterações vocais: revisão sistemática
}

\author{
Quality of life and anxiety related to \\ voice disorders: Systematic review
}

\author{
Chenia Caldeira MARTINEZ \\ Léia Gonçalves GURGEL² \\ Rodrigo Della Méa PLENTZ ${ }^{3}$ \\ Caroline Tozzi REPPOLD ${ }^{2}$ \\ Mauriceia CASSOL $^{4}$
}

\begin{abstract}
Resumo
Este estudo objetivou revisar sistematicamente na literatura as relações entre a disfonia e a ansiedade e qualidade de vida. Como estratégia de busca, foram utilizados os termos "ensaio clínico randomizado", "ansiedade", "voz", "distúrbios da voz", "qualidade da voz" e "treinamento da voz", nas bases de dados MedLine (PubMed), Cochrane Central, Embase, PsycINFO, Lilacs e SciELO. Incluíram-se ensaios clínicos randomizados de fonoterapia vocal com os desfechos ansiedade e qualidade de vida. Utilizou-se a abordagem Grades of Recommendation, Assessment, Development and Evaluation, que tem o objetivo de determinar a qualidade da evidência para cada desfecho. Dos 137 estudos identificados, apenas três atenderam aos critérios de inclusão e foram considerados como relevantes para a composição da amostra deste trabalho. A maioria dos estudos encontrados apresentavam limitações metodológicas, resultados imprecisos e ausência de razão de chances. Dentre as limitações desta revisão, destaca-se que parte dos achados esteve relacionada ao autorrelato do paciente e que dois ensaios foram realizados com a mesma amostra. Apesar do pequeno número de estudos, constata-se que a ansiedade e a qualidade de vida estão associadas à disfonia.
\end{abstract}

Palavras-chave: Ansiedade; Disfonia; Qualidade de vida; Voz.

\begin{abstract}
The aim of the study was to systematically review published literature about the relationship between dysphonia, anxiety and quality of life. The research strategy was to search for the keywords "randomized clinical trial", "anxiety", "voice", "voice disorders", "voice quality", "voice training" in the literature databases of MedLine (PubMed), Cochrane Central, Embase, PsycINFO, Lilacs, SciELO. We included randomized clinical trials of voice treatment involving anxiety

1 Prefeitura Municipal de Tramandaí, Secretaria Municipal de Educação e Cultura. Tramandaí, RS, Brasil.

2 Universidade Federal de Ciências da Saúde de Porto Alegre, Programa de Pós-Graduação em Ciências da Saúde. Rio Grande do Sul, RS, Brasil.

3 Universidade Federal de Ciências da Saúde de Porto Alegre, Programa de Pós-Graduação em Ciências da Saúde, e Programa de Pós-Graduação em Ciências da Reabilitação. Rio Grande do Sul, RS, Brasil.

${ }^{4}$ Universidade Federal de Ciências da Saúde de Porto Alegre, Curso de Fonoaudiologia, Departamento de Fonoaudiologia. R. Sarmento Leite, 245, 90050-170, Porto Alegre, RS, Brasil. Correspondência para/Correspondence to: M. CASSOL. E-mail: <m.cassol@terra.com.br>.
\end{abstract}


and quality of life outcomes. The GRADE approach was used, which aims to determine the quality of evidence for each outcome. Of 137 studies identified, three were included according to our inclusion and exclusion criteria. Most of the studies included presented methodological limitations, inaccurate results and absence of odds ratio. Among the limitations of this study, some findings were related to the patient's self-report and two studies were performed with the same sample. Despite the small number of studies, it is found that anxiety and quality of life are associated with dysphonia.

Keywords: Anxiety; Dysphonia; Quality of life; Voice.

A produção da voz é um processo complexo que envolve o controle de vários mecanismos reguladores (Madeira \& Tomita, 2010), que irão organizar, planejar e executar, de forma coordenada, os movimentos dos músculos envolvidos nesse ato motor (Lopes Filho, Campiotto, Levy, Redondo, \& Anelli-Bastos, 2005). Embora a fonação seja uma função neurofisiológica inata, ela se desenvolve em paralelo com o desenvolvimento orgânico e psicológico do indivíduo (Behlau, 2008), podendo ser considerada uma manifestação psicológica com sofisticado processamento muscular (Lopes Filho et al., 2005; Behlau, 2008).

Déficits na produção vocal são caracterizados como disfonia, em que a voz não cumpre seu papel básico de transmissão da mensagem verbal e emocional do indivíduo. Pesquisas revelam que o diagnóstico da disfonia pode estar diretamente relacionado ao estresse, ansiedade, depressão, introversão e fobias sociais (Almeida, Behlau, \& Leite, 2011; Seifert \& Kollbrunner, 2005). O estudo sobre a relação entre a voz e os sintomas psicológicos indica que uma alteração vocal pode comprometer a qualidade da comunicação e das relações sociais do indivíduo, afetando diretamente sua qualidade de vida (Spina, Maunsell, Sandalo, Gusmão, \& Crespo, 2009).

A ansiedade é um estado emocional que tem componentes fisiológicos e comportamentais, e pode ocasionar incapacidade funcional (Ravindran \& Stein, 2010). Essa desordem possui diversos fatores de risco e comorbidades (Borges \& Angelotti, 2002; Conway, Compton, Stinson, \& Grant, 2006; Meng \& D'arcy, 2012), abrangendo diversos sintomas psicológicos, somáticos e sinestésicos (Guidolin \& Celia, 2011; B.J. Sadock \& Sadock, 2007). Os sintomas de ansiedade têm se tornado uma evi-

512 dente preocupação de caráter ocupacional, consti- tuindo um importante fator de risco para a saúde humana (M. Angelillo, Di Maio, Costa, Angelillo, \& Barillari, 2009; Araújo et al., 2008; Thibeault, Merrill, Roy, Gray, \& Smith, 2004).

Nesse contexto, estudos apontam que a produção da voz pode ser afetada pelo estado emocional e psicológico do indivíduo (Kotby, Baraka, Sady-Ghanen, \& Shoeib, 2003). No entanto, as doenças vocais também afetam severamente o bem-estar e a saúde dos pacientes disfônicos, causando redução da qualidade de vida e aumento nos escores de depressão (Cohen, 2010; Ma \& Yiu, 2001; Thibeault et al., 2004). A relação entre esses processos é complexa, e a influência de cada fator é manifestada de forma heterogênea e particular (Kollbrunner, Menet, \& Seifert, 2010; Mackenzie, Millar, Wilson, Sellars, \& Deary, 2001).

É clara a necessidade do desenvolvimento de pesquisas na área, que visem à identificação e compreensão da relação entre alterações vocais e sintomas emocionais, como a ansiedade. Ainda, é preciso que esses estudos se apresentem em situações ideais de pesquisa, como ensaios clínicos controlados randomizados (Escosteguy, 1999; Higgins, Thompson, Deeks, \& Altman, 2003). Esse trabalho teve o objetivo de revisar sistematicamente, na literatura, quais as relações entre a disfonia e a ansiedade e qualidade de vida dos pacientes disfônicos que estão em avaliação ou tratamento vocal.

\section{Método}

\section{Procedimentos}

Para a busca, foram consultadas as bases de dados eletrônicas Medline (via PubMed), Embase, 
Cochrane Central, Psyclnfo, Lilacs e SciELO, além de busca manual. As palavras-chave utilizadas foram "ensaio clínico randomizado", "ansiedade", "voz", "distúrbios da voz", "qualidade da voz" e "treinamento da voz". Conforme a base de dados, foram utilizados descritores correspondentes nos idiomas português, inglês e espanhol. Foi abrangido o período entre janeiro de 1980 e janeiro de 2013. Referências adicionais foram consultadas, e não houve nenhuma restrição quanto ao termo "ansiedade". O desfecho primário é a ansiedade, e o desfecho secundário é a qualidade de vida desses pacientes. Por ser secundário, optou-se por não utilizar o descritor "quality of life" na busca. Ainda, com o uso desse descritor, as buscas ficariam extremamente restritas e não reproduziriam o real estado da arte da relação entre disfonia, ansiedade e qualidade de vida.

Foram incluídos todos os estudos com delineamento do tipo ensaio clínico controlado randomizado, sem restrição de idioma, que relacionassem a voz ao sintoma de ansiedade, e que incluíssem indivíduos com alteração vocal autorrelatada ou avaliada por examinador. De outro lado, foram excluídos todos os estudos que não estabelecessem de maneira confiável uma definição do construto ansiedade, de intervenção em voz, ou que não incluíssem indivíduos com alterações vocais.

Os títulos e os resumos de todos os artigos foram avaliados de forma independente e cegada. Os resumos que não forneceram informações suficientes em relação aos critérios de inclusão e exclusão foram selecionados para avaliação do texto integral, conforme os critérios de elegibilidade. Foram coletadas informações referente às características metodológicas, intervenções e desfechos dos estudos, por meio de formulários padronizados. Todas as fases do estudo foram realizadas por duas revisoras independentes - fonoaudióloga e psicóloga -, devidamente treinadas para tal fim, sendo as discordâncias resolvidas por consenso.

\section{Instrumentos}

A qualidade dos estudos foi avaliada com base nos seguintes aspectos principais: sigilo da lista de alocação; análise por intenção de tratar; comparabilidade basal; cegamento da avaliação dos desfechos; e descrição das perdas e exclusões. A falta de uma descrição sobre como foi gerada a lista de alocação (o que poderia incluir termos como "central", "web-based" ou "telephone randomization", ou uma clara declaração de que a lista de alocação foi sigilosa) foi considerada como ausência do sigilo de alocação. A única possibilidade de cegamento nesse tipo de estudo reside na avaliação dos desfechos, de tal modo que a falta da descrição desse tipo de situação caracterizava a pesquisa como um estudo aberto.

A qualidade dos estudos também foi avaliada por meio da abordagem Grades of Recommendation, Assessment, Development and Evaluation (GRADE), recomendada pela Colaboração Cochrane (Guyatt et al., 2008). Para cada resultado, a qualidade da evidência foi baseada em cinco fatores: (1) limitações na metodologia dos estudos; (2) consistência dos resultados; (3) subjetividade; (4) precisão; e (5) potencial para viés de publicação. A qualidade foi reduzida em um nível para cada fator não satisfeito. A abordagem GRADE resulta em quatro níveis de qualidade de evidências: alta, moderada, baixa e muito baixa (Guyatt et al., 2008).

A análise dos dados foi descritiva quanto à qualidade de vida e aos sintomas de ansiedade, bem como à relação destes com a voz. Foram relatadas as características metodológicas e as principais evidências, de acordo com o objetivo central das pesquisas.

\section{Resultados}

Como resultado da busca inicial, puderam ser identificados 137 resumos. Destes, três atenderam aos critérios de inclusão e foram considerados como relevantes para a composição da amostra deste trabalho (Figura 1).

Todos os estudos incluídos na presente revisão consideraram os desfechos ansiedade e qualidade de vida. De acordo com a abordagem GRADE, a qualidade da evidência dos estudos selecionados foi baixa, em razão das limitações na metodologia, 
inconsistência dos resultados, imprecisão e ausência de razão de chances nos estudos (Tabela 1).

Quanto aos periódicos em que os estudos foram publicados, constata-se que todos são indexados na base de dados do ISI Web of Knowledge e apresentaram fator de impacto que variou de 0.400 a 14.093 (Tabela 2). Na Tabela 2, podem ser observadas as características dos ensaios incluídos.
O estudo A (Mackenzie et al., 2001) relacionou ansiedade e fonoterapia da voz. Os participantes, 204 disfônicos, foram submetidos a avaliação videolaringoscópica, análise perceptivoauditiva e acústica, questionário de perfil vocal, Hospital Anxiety and Depression Scale (HADS, Escala Hospitalar de Ansiedade e de Depressão) (Zigmond \& Snaith, 1983), entrevista e SF-36 (instrumento que avalia saúde geral e qualidade de vida)

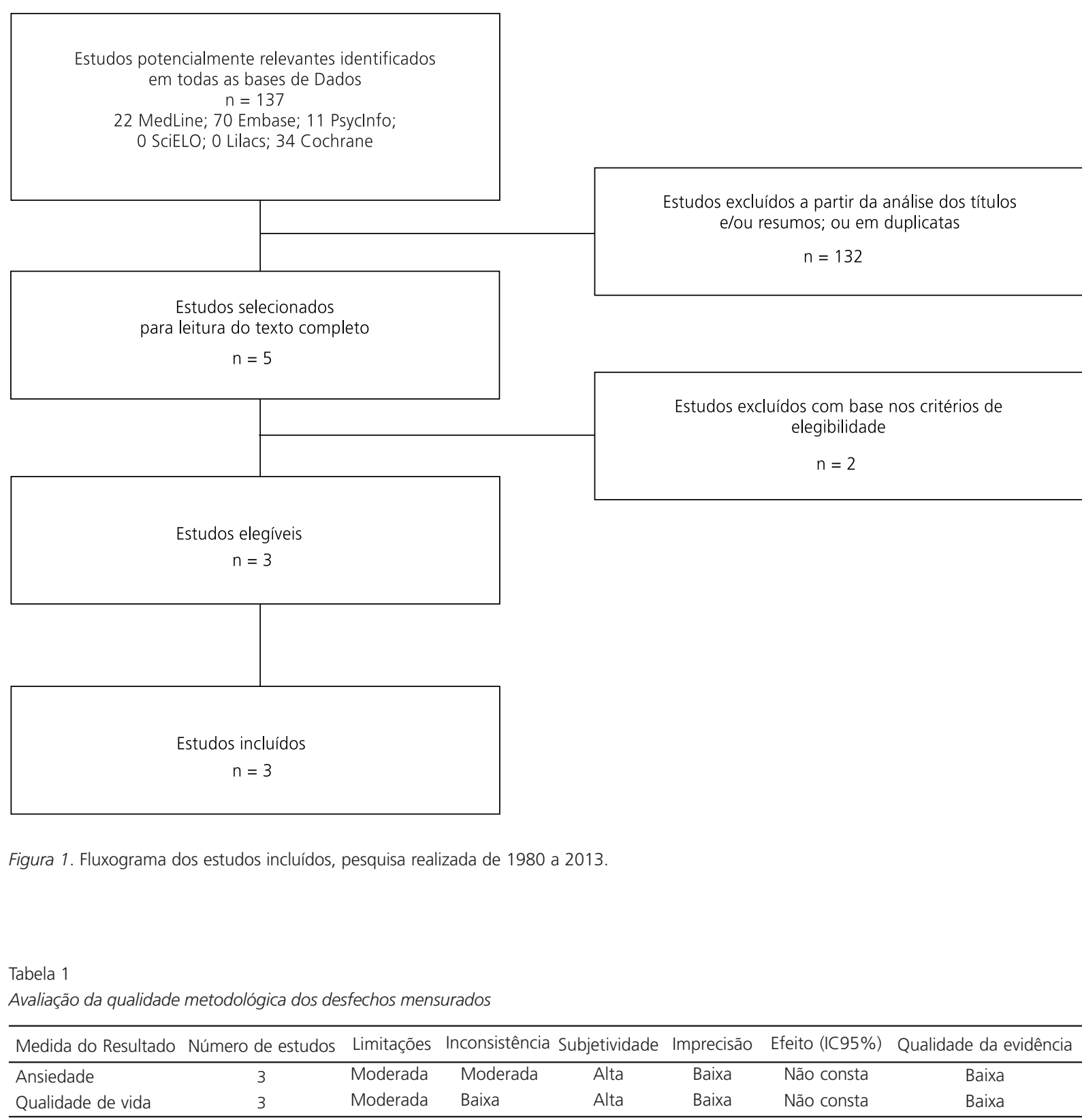

Nota: IC $95 \%$ : Intervalo de Confiança de $95 \%$. 
Tabela 2

Características dos estudos incluídos nesta revisão, realizada de 1980 a 2013

\begin{tabular}{llll}
\hline \multirow{2}{*}{ Características } & \multicolumn{2}{c}{ Estudo } \\
\cline { 2 - 4 } & A & B & C \\
\hline Autor (ano) & Mackenzie et al. (2001) & Deary et al. (2003) & Pülz et al. (2009) \\
Periódico (Fl) & BMJ (14.093) & Clin. Otolaryngol (2.393) & HNO (0.400) \\
Língua original & Inglês & Inglês & Alemão \\
País de origem & Reino Unido & Reino Unido & Alemanha \\
Tipo de estudo & ECR & ECR & ECR \\
Pacientes & Disfônicos & Disfônicos & Disfônicos \\
Idade da amostra & $>16$ anos & Média de 52 anos \pm 15 anos & Média de 51,1 anos \pm 16,4 anos \\
Sexo & 51 (masculino),153 (feminino) & 51 (masculino),153 (feminino) & 21 (masculino),33 (feminino) \\
Intervenção & Terapia vocal & Avaliação da voz e fatores psicológicos & Avaliação psicométrica da motivação com a \\
& & & terapia \\
Desfecho & Ansiedade; qualidade de vida & Ansiedade; qualidade de vida & Ansiedade; qualidade de vida \\
\hline
\end{tabular}

Nota: FI: Fator de Impacto; BMJ: British Medical Journal; ECR: Ensaio Clínico Randomizado.

(Mchorney, Ware, \& Raczek, 1993). A fonoterapia englobou técnicas de orientação e treinamento vocal, conforme a necessidade individual. Concluiu-se que a terapia vocal é eficaz para a disfonia, avaliada pelo autorrelato do paciente e do avaliador $(F=10.96 ; p=0.001)$. Esses pacientes apresentaram maior queixa de sintomas psicológicos e menor qualidade de vida quando comparados com indivíduos sem disfonia. A intervenção teve efeito significativo na qualidade de vida (avaliada pelo SF-36), no parâmetro saúde mental $(F=6.08 ; p=0.02)$.

O estudo B (Deary, Wilson, Carding, \& Mackenzie, 2003) relacionou ansiedade e avaliação da voz em uma amostra de 204 disfônicos com sintomas psicológicos. Foram correlacionadas medidas de autorrelato do paciente (Vocal Performance Questionnaire) (Carding \& Horsley, 1992), avaliação vocal pelo terapeuta (Buffalo Voice Profile Scale) (Wilson, 1991) e análise acústica da voz. Esses três índices de qualidade vocal foram comparados com diferenças individuais em instrumentos que avaliam sintomas psicológicos, dentre os quais a ansiedade e a qualidade de vida. Foram observadas correlações significativas entre os problemas descritos no autorrelato do paciente. Os pacientes que relataram mais problemas vocais apresentaram mais alterações psicológicas e pior qualidade de vida. A classificação da voz, realizada por especialistas, foi correlacionada $(p<0,01)$ com distúrbio neurótico e qualidade de vida.
O estudo C (Pulz et al., 2009) observou a interferência da ansiedade e da terapia psicológica na motivação/satisfação do paciente que está em avaliação e tratamento vocal. Foram randomizados 54 disfônicos em dois grupos, ambos submetidos à avaliação da voz (perceptivo-auditiva e acústica), da qualidade de vida em voz (Hogikyan \& Sethuraman, 1999) e da ansiedade e depressão (Escala Gießener Beschwerdebogen) (E. Brähler, Schumacher, \& Brähler, 2000). Um grupo respondeu aos questionários antes da consulta médica, quando o paciente seria confrontado em suas respostas e sobre a motivação quanto ao tratamento. O outro grupo realizou a avaliação da voz e esclarecimento sobre o tratamento; somente após, o paciente respondia aos questionários, sem ter suas respostas confrontadas. Os autores concluíram que essa inter-venção afeta positivamente a motivação do paciente disfônico, ressaltando também a importância de sua relação com o terapeuta.

A extração e a análise dos dados referente à qualidade metodológica dos ensaios incluídos foram realizadas por dois investigadores independentes. Esses achados estão apresentados na Tabela 3.

Os resultados da presente revisão apontaram que dois dos estudos encontrados tiveram como foco a relação entre a intervenção fonoaudiológica e a ansiedade (Mackenzie et al., 2001; Deary et al., 2003), e que apenas um dos estudos focalizou a 
Tabela 3

Qualidade metodológica dos estudos incluídos

\begin{tabular}{|c|c|c|c|c|c|c|}
\hline Estudo & Randomização & Sigilo de alocação & $\begin{array}{c}\text { Análise por intenção } \\
\text { de tratar }\end{array}$ & Comparabilidade basal & Cegamento & $\begin{array}{c}\text { Descrição de } \\
\text { perdas e exclusões }\end{array}$ \\
\hline Mackenzie et al., 2001 & 1 & 1 & I & $A$ & $A$ & A \\
\hline Deary et al., 2003 & I & I & I & $A$ & I & A \\
\hline Pülz et al., 2009 & 1 & I & I & 1 & 1 & I \\
\hline
\end{tabular}

Nota: A: Adequado; I: Inadequado.

intervenção psicológica em pacientes disfônicos (Pulz et al., 2009). Dos três ensaios incluídos, nenhum apresentou relação estatisticamente significativa entre alterações vocais e sintoma de ansiedade; porém esses construtos estiveram sempre relacionados, demonstrando que alterações na voz e sintomas psicológicos são fatores que podem estar interligados. Quanto à qualidade de vida, encontrou-se relevância referente ao aspecto da saúde mental.

A fonoterapia pode reduzir os sintomas psicológicos, incluindo a ansiedade (Mackenzie et al., 2001). Estudos revelam a interferência da ansiedade na forma de expressão e comunicação do indivíduo, seja em relação ao corpo, à fala e/ou à voz (Almeida et al., 2011; Seifert \& Kollbrunner, 2005; Dietrich, Abbott, Gartner-Schmidt, \& Rosen, 2008), seja também o impacto da disfonia na qualidade de vida do paciente (Cohen, 2010). Quanto maior o grau de ansiedade, maior poderá ser o comprometimento na comunicação e na qualidade de vida, bem como o número de sinais e sintomas vocais referidos pelos indivíduos (Almeida et al., 2011).

Em um dos estudos (Deary et al., 2003), as associações mais fortes de variáveis psicológicas e de medidas vocais estão relacionadas ao autorrelato do disfônico. Isso sugere que é a percepção dos pacientes sobre sua própria qualidade de voz que, de fato, está associada aos fatores psicológicos em indivíduos com sintomas vocais. Esse estudo, assim como outro (Kotby et al., 2003), refere que a produção da voz está sujeita aos estados psicológicos pelos quais o indivíduo passa ao longo da vida.

Há controvérsias na literatura sobre a relação

516 entre a percepção da qualidade vocal e seus im- pactos na qualidade de vida, medida pelo autorrelato dos disfônicos e pela avaliação de profissionais da área. De fato, a voz pode ter um impacto negativo na qualidade de vida, porém esses impactos frequentemente não estão correlacionados, de forma significativa, com o diagnóstico da doença laríngea ou com o grau de alteração da voz (Queija et al., 2007; Bassi et al., 2011), corroborando os resultados de Deary et al. (2003). Atentando para a percepção que o disfônico tem da sua alteração vocal (Hummel, Scharf, Schuetzenberger, Graessel, \& Rosanowski, 2010), será possível oferecer intervenções interdisciplinares que poderão otimizar resultados terapêuticos (Defina, Massih, \& Mamede, 2004; Meulenbroek, Van Opstal, Claes, Marres, \& De Jong, 2012).

Quanto à amostra dos estudos incluídos na presente revisão, observa-se que todos os ensaios foram compostos por homens e mulheres, sendo sempre o número destas mais elevado. Essa constatação revela a maior prevalência de alterações vocais em indivíduos do gênero feminino, conforme já descrito na literatura (Angelillo et al., 2009; Hummel et al., 2010; Van Houtte, Claeys, Wuyts, \& Van Lierde, 2011).

A fim de complementar esta pesquisa, foram avaliados de maneira específica outros parâmetros metodológicos, importantes para a definição dos resultados. Apenas o estudo A apresentou a descrição do cegamento (simples cego), do sigilo da lista de alocação (sequência aleatória gerada pelo computador) e das perdas e exclusões ao longo da pesquisa. A análise por intenção de tratar e a comparabilidade basal não foram especificadas em nenhum dos estudos. Nesse sentido, o estudo A apresentou-se como de melhor qualidade em comparação aos demais. 
Ressalta-se que dois dos estudos incluídos foram realizados com a mesma amostra e pelos mesmos autores, enfocando cada qual níveis de intervenção diferentes: avaliação e tratamento (Deary et al., 2003; Mackenzie et al., 2001). Isso evidencia a escassez de estudos que investigam o tema em situações ideais de pesquisa. Ainda, dentre as limitações desta revisão, destaca-se que a maioria dos trabalhos incluídos estiveram relacionados ao autorrelato do paciente, não incluindo avaliação global da voz feita por um especialista na área. Esse pode ter sido um fator causador de viés nos resultados obtidos pelos estudos.

Observa-se que existem na literatura poucos estudos que abordem a relação entre as características vocais e psicológicas de pacientes disfônicos. Apesar disso, esta revisão mostrou que a ansiedade e a qualidade de vida estão associadas às alterações vocais, especialmente quando é abordado o autorrelato do paciente.

A produção de novos estudos, com maior número amostral e melhor qualidade metodológica, é fundamental para aprofundar a compreensão das implicações das alterações vocais na ansiedade e na qualidade de vida. Destaca-se a importância desses estudos para o melhor conhecimento da relação entre os aspectos vocais e psicológicos, com o objetivo de se criarem programas de tratamento mais adequados, que contribuam para o enfrentamento dos casos clínicos com tais características. Ressalta-se, por fim, a importância da interdisciplinaridade entre as áreas da saúde, complementando o tratamento do paciente.

\section{Referências}

Almeida, A. A. F., Behlau, M., \& Leite, J. R. (2011). Correlação entre ansiedade e performance comunicativa. Revista da Sociedade Brasileira de Fonoaudiologia, 16(4), 384-389.

Angelillo, M., Di Maio, G., Costa, G., Angelillo, N., \& Barillari, U. (2009). Prevalence of occupational voice disorders in teachers. Journal of Preventive Medicine and Hygiene, 50(1), 26-32.

Araújo, T. M., Reis, E. J. F. B., Carvalho, F. M., Porto, L. A., Reis, I. C., \& Andrade, J. M. (2008). Fatores associados a alterações vocais em professoras. Cadernos de Saúde Pública, 24(6), 1229-1238.
Bassi, I. B., Assunção, A. A., De Medeiros, A. M., De Menezes, L. N., Teixeira, L. C., \& Côrtes Gama, A. C. (2011). Quality of life, self-perceived dysphonia, and diagnosed dysphonia through clinical tests in teachers. Journal of Voice, 25(2), 192-201.

Behlau, M. (2008). Voz: o livro do especialista. Rio de Janeiro: Revinter.

Borges, N. B, \& Angelotti, G. S. (2002). Ansiedade e depressão em uma amostra de pacientes classificados como portando fatores psicológicos que afetam as condições médicas. Estudos de Psicologia (Campinas), 19(3),15-22. http://dx.doi.org/10.1590/S0103-166X2 002000300002

Brähler, E., Schumacher, J., \& Brähler, C. (2000). First all-Germany standardization of the brief form of the Gissen Complaints Questionnaire GBB-24. Psychotherapie, Psychosomatik, Medizinische, Psychologie, 50(1), 14-21.

Carding, P. N., \& Horsley, I. A. (1992). An evaluation of voice therapy in non-organic dysphonia. European Journal of Disorders of Communication, 27(2), 137-158.

Cohen, S. M. (2010). Self-reported impact of dysphonia in a primary care population: An epidemiological study. Laryngoscope, 120(10), 2022-2032.

Conway, K. P., Compton, W., Stinson, F. S., \& Grant, B. F. (2006). Lifetime comorbidity of DSM-IV mood and anxiety disorders and specific drug use disorders: Results from the National Epidemiologic Survey on Alcohol and Related Conditions. The Journal of Clinical Psychiatry, 67(2), 247-257.

Deary, I. J., Wilson, J. A., Carding, P. N., \& Mackenzie, K. (2003). The dysphonic voice heard by me, you and it: Differential association with personality and psychological distress. Clinical Otolaryngology and Allied Science, 28(4), 374-378.

Defina, A. P., Massih, D. A., \& Mamede, R. C. M. (2004). Report of experience of performance of speech language pathologist and psychology with head and neck patient's cancer. Revista Brasileira de Cirurgia de Cabeça e Pescoço, 33(1), 45-48.

Dietrich, M., Abbott, K. V., Gartner-Schmidt, J., \& Rosen, C. A. (2008). The frequency of perceived stress, anxiety, and depression in patients with common pathologies affecting voice. Journal of Voice, 22(4), 472-488.

Escosteguy, C. C. (1999). Tópicos metodológicos e estatísticos em ensaios clínicos controlados randomizados. Arquivos Brasileiros de Cardiologia, 72(2), 139-143.

Guidolin, B. L., \& Celia, S. A. H. (2011). Sintomas depressivos e de ansiedade em mães durante internação pediátrica em um hospital universitário. Revista de Psiquiatria do Rio Grande do Sul, 33(2), 80-86.

Guyatt, G. H., Oxman, A. D., Vist, G. E., Kunz, R., FalckYtter, Y., Alonso-Coello, P., \& Schünemann, P. (2008). GRADE: An emerging consensus on rating quality of 
evidence and strength of recommendations. British Medical Journal, 336(7650), 924-926.

Higgins, J. P. T, Thompson, S. G., Deeks, J. J., \& Altman, D. G. (2003). Measuring inconsistency in meta-analyses. British Medical Journal, 327(7414), 557-60.

Hogikyan, N. D., \& Sethuraman, G. (1999). Validation of an instrument to measure Voice-Related Quality of Life (V-RQOL). Journal of Voice, 13(4), 557-569.

Hummel, C., Scharf, M., Schuetzenberger, A., Graessel, E., \& Rosanowski, F. (2010). Objective voice parameters and self-perceived handicap in dysphonia. Folia Phoniatrica et Logopaedica, 62(6), 303-307.

Kollbrunner, J., Menet, A., \& Seifert, E. (2010). Psychogenic aphonia: No fixation even after a lengthy period of aphonia. Swiss Medical Weekly, 140(1-2), 12-17.

Kotby, M. N., Baraka, M., Sady-Ghanen, M., \& Shoeib, R. (2003). Psychogenic stress as a possible etiological factor in non-organic dysphonia. Proceedings of the XVII World Congress of the International Federation of Oto-Rhino-Laryngological Societies, 1240(1-2), 1251-1256.

Lopes Filho, O., Campiotto, A. R., Levy, C., Redondo, M. C., \& Anelli-bastos, W. (2005). Tratado de fonoaudiologia ( $2^{\mathrm{a}}$ ed.). Ribeirão Preto: Tecmedd.

Ma, E. P., \& Yiu, E. M. (2001). Voice activity and participation profile: Assessing the impact of voice disorders on daily activities. Journal of Speech, Language, and Hearing Research, 44(3), 511-524.

Mackenzie, K., Millar, A., Wilson, J. A., Sellars, C. S., \& Deary, I. J. (2001). Is voice therapy an effective treatment for dysphonia? A randomised controlled Trial. British Medical Journal, 323, 1-6.

Madeira, F. B., \& Tomita, S. (2010). Voice handicap index evaluation in patients with moderate to profound bilateral sensorineural hearing loss. Brazilian Journal of Otorhinolaryngology, 76(1), 59-70.

Mchorney, C. A., Ware, L. U., \& Raczek, A. E. (1993). The MOS-36: Item short-form health survey (SF-36): II psychometric and clinical tests of validity in measuring physical and mental health constructs. Medical Care, 31(3), 247-263.

Meng, X., \& D'Arcy, C. (2012). Common and unique risk factors and comorbidity for 12-month mood and anxiety disorders among canadians. Canadian Jornal of Psychiatry, 57(8), 479-487.
Meulenbroek, L. F., Van Opstal, M. J., Claes, L., Marres, H. A., \& De Jong, F. I. (2012). The impact of the voice in relation to psychosomatic well-being after education in female student teachers: A longitudinal, descriptive study. Journal of Psychosomatic Research, 72(3), 230-235.

Pülz, S., Schützenberger, A., Grässel, E., Köllner, V., Eysholdt, U., \& Rosanowski, F. (2009). Satisfaction of dysphonic patients with psychosomatic assessment and intervention. HNO, 57(9), 902-909.

Queija, D. S., Ferreira, A. S., Portas, J. G., Dedivitis, R. A., Pfuetzenreiter Junior, E. G., Bohn, N. P., \& Barros, A. P. B. (2007). Avaliação vocal e auto-percepção da desvantagem vocal (VHI) após laringectomia fronto-lateral. Revista Brasileira de Cirurgia de Cabeça e Pescoço, 36(2), 95-99.

Ravindran, L. N., \& Stein, M. B. (2010). The pharmacologic treatment of anxiety disorders: A review of progress. The Journal of Clinical Psychiatry, 71(7), 839-854.

Sadock, B. J., \& Sadock, V. A. (2007). Compêndio de psiquiatria: ciência do comportamento e psiquiatria clínica ( $9^{a}$ ed.). Porto Alegre: Artmed.

Seifert, E., \& Kollbrunner, J. (2005). Stress and distress in non-organic voice disorder. Swiss Medical Weekly, 135(27-28), 387-397.

Spina, A. L., Maunsell, R., Sandalo, K., Gusmão, R., \& Crespo, R. (2009). Correlação da qualidade de vida e voz com atividade profissional. Revista Brasileira de Otorrinolaringologia, 75(2), 275-279.

Thibeault, S., Merrill, R. M., Roy, N., Gray, S. D., \& Smith, E. M. (2004). Occupational risk factors associated with voice disorders among teachers. Annals of Epidemiology, 14(10), 786-792.

Van Houtte, E., Claeys, S., Wuyts, F., \& Van Lierde, K. (2011). The impact of voice disorders among teachers: Vocal complaints, treatment-seeking behavior, knowledge of vocal care, and voice-related absenteeism. Journal of Voice, 25(5), 570-575.

Wilson, D. K. (1991). Problemas de voz em crianças. São Paulo: Manole.

Zigmond, A. S., \& Snaith, R. P. (1983). The Hospital Anxiety and Depression Scale. Acta Psychiatrica Scandinavica, 67(6), 361-370.

Recebido: junho 25, 2013

Versão final: março 26, 2014

Aprovado: abril 25, 2014 